R. Kaufman

Nagoya Math. J.

Vol. 56 (1974), 139-145

\title{
LARGE INCREMENTS OF BROWNIAN MOTION
}

\author{
R. KAUFMAN
}

1. Let $X(t)$ denote Brownian motion on the line $0 \leq t<\infty$, let $g(h)=\left(2 h \log h^{-1}\right)^{1 / 2}$, and let $0<\alpha<1$. Orey and Taylor [5] have investigated the random set defined by the inequalities

$$
E_{\alpha}: 0 \leq t \leq 1, \lim \sup X(t+h)-X(t) / g(h) \geq \alpha
$$

and proved that $P\left\{\operatorname{dim} E_{\alpha}=1-\alpha^{2}\right\}=1$. Here we prove two theorems on $E_{\alpha}$ that reflect more subtle properties of $E_{\alpha}$ than its Hausdorff dimension alone.

THEOREM 1. With probability 1, a certain compact subset of $E_{\alpha}$ carries a probability measure $\mu$ such that $\hat{\mu}(u)=o\left(u^{\frac{1}{2}\left(\alpha^{2}-1\right)}\right), 1 \leq u<\infty$.

THEOREM 2. Let $F$ be a closed set in $(0,1)$ of dimension $d \geq \alpha^{2}$. Then

$$
P\left\{\operatorname{dim} F \cap E_{\alpha} \geq d-\alpha^{2}\right\}=1 .
$$

For every pair $d, \alpha$ with $1>d \geq \alpha^{2}$, there is almost-sure equality for a certain fixed set $F_{1}$ of dimension $d$. For every $\alpha$ there is a set $F_{2}$ of dimension $1-\alpha^{2}$ such that $\operatorname{dim} F_{2} \cap E_{\alpha}=1-\alpha^{2}$ almost surely.

The standard reference concerning relations between Fourier-Stieltjes transforms and Hausdorff measures is [3]: in particular, by a theorem of Beurling [3, Ch. III], the property of $E_{\alpha}$ claimed in Theorem 1 is stronger than the lower bound on $\operatorname{dim} E_{\alpha}$ found by Orey and Taylor. For example, by a theorem of Zygmund $[1$, p. 413;6] the property of $E_{\alpha}$ is not even shared by certain sets of positive Lebesgue measure. Further examples concerning dimension and Fourier analysis are presented in [2], theorems on Brownian motion and dimension in [4 a - d], while the indeterminacy of intersections of random sets and fixed sets (as in the second and third statements in Theorem 2) was observed in [4e].

Received March 26, 1973. 
Thanks are due to Orey and Taylor for a preprint of [5] and to $N$. Jain for pointing out many obscurities.

2. In the proofs we need estimates for sums $\sum\left(p-\xi_{n}\right) a_{n}$, where the $\xi_{n}$ are independent random variables with common distribution

$$
P\left\{\xi_{n}=1\right\}=p=1-P\left\{\xi_{n}=0\right\} ; \sigma^{2}=\sum\left|a_{n}\right|^{2},
$$

$B=\max \left|a_{n}\right|$. The basic inequality is

$$
p e^{t(1-p)}+(1-p) e^{-p t} \leq 1+p(1-p) t^{2} \leq \exp t^{2} p,
$$

valid for $0 \leq p \leq 1,-1 \leq t \leq 1$. In case the coefficients are real, we find by Chebyshev's inequality

$$
P\left\{\left|\sum\right| \geq Y\right\} \leq 2 \exp t^{2} p \sigma^{2} \exp -t Y, \quad 0 \leq t B \leq 1 .
$$

Choosing the best value of $t$ we find

$$
P\left\{\left|\sum\right| \geq Y\right\} \leq 2 \exp -1 / 4 p^{-1} \sigma^{-2} Y^{2}, \quad \text { provided } Y B \leq 2 p \sigma^{2} .
$$

In the case of complex numbers $a_{n}$ in the sum $\sum\left(p-\xi_{n}\right) a_{n}$, we have merely to replace $Y$ by $\frac{1}{2} Y$ and double the bounds so obtained; this estimate is rough, but sufficient.

3. Let $S$ be the functional $\max |X(b)-X(a)|(0 \leq a<b \leq 1)$; we need only the 'tail' of the distribution of $S$, namely

$$
P\{S \geq Y\}=\exp -\frac{1}{2} Y^{2} \exp o\left(Y^{2}\right), \quad Y \rightarrow+\infty .
$$

This estimate is obtained simply from the Gaussian law and the reflection principle, and is of course valid for $P\{X(1)-X(0) \geq Y\}$. We use it now to obtain an estimate from [5], involving parameters $0<\beta \leq 1$, $0<b<1$. The event

$$
X(h)-X(0) \geq \beta g(h),|X(t)-X(0)| \leq 2 b^{\frac{1}{2}} g(h) \quad \text { on }[0, b h]
$$

has probability $h^{\beta^{2}} h^{o(1)}-o(h)$ as $h \rightarrow 0+$. Thus the event

$$
X(h)-X(t) \geq\left(\beta-2 b^{\frac{1}{2}}\right) g(h) \quad \text { on } 0 \leq t \leq b h
$$

has probability $>h^{\beta^{2}} h^{o(1)}$ for small $h>0$. With the aid of this inequality we can begin to construct the measure $\mu$. Let $0 \leq r<s \leq 1$ and let $I_{n}(1 \leq n \leq N)$ be the usual division of $(r, s)$ into adjacent intervals of length $(s-r) N^{-1}$; supposing that $b^{-1}$ is an integer (as in [5]) we have a further subdivision of each $I_{n}$ into intervals $I_{n}^{q}\left(1 \leq q \leq \mathrm{b}^{-1}\right)$ of length 
$(s-r) b N^{-1}$. An interval $I_{n}^{q}$ with left extremity $x$ is selected if

$$
X(x+h)-X(t) \geq\left(\beta-2 b^{\frac{1}{2}}\right) g(h) \quad \text { on } x \leq t \leq x+b h,
$$

with $h=N^{-1}(s-r)$. We put $\beta_{1}=\beta-2 b^{\frac{1}{2}}$ and suppose that $0<\beta_{1}<\beta$ $<\alpha^{2}$. The selections of the intervals $I_{n}^{q}(1 \leq n \leq N)$ are mutually independent for each $q$, with a probability $p=p_{N} \geq N^{-\beta^{2}} N^{o(1)}$, for large $N$. Let $m_{0}$ be Lebesgue measure in $(r, s)$, let $\xi$ be the characteristic function of the selected intervals, and let $m_{1}(d x)=p^{-1} \xi(x) m_{0}(d x)$.

LEMma. For any $\varepsilon>0$ the inequality $\left|\hat{m}_{1}(u)-\hat{m}_{0}(u)\right|<\varepsilon(1+u)^{\frac{1}{2}\left(\alpha^{2}-1\right)}$ for all $u>0$ holds, with probability approaching 1 as $N \rightarrow \infty$.

Proof. The parameter $q=1, \cdots, b^{-1}$ determines decompositions $m_{1}$ $=\sum m_{1}^{q}$ and $m_{0}=\sum m_{0}^{q}$; because $b$ is fixed it is sufficient to prove the inequality for each pair $\hat{m}_{0}^{q}$ and $\hat{m}_{1}^{q}$, as we now do, dropping the superscript $q$. Now $\hat{m}_{0}(u)-\hat{m}_{1}(u)=\sum\left(1-p^{-1} \xi_{n}\right) f_{n}(u)$ where

$$
\left|f_{n}\right| \leq b N^{-1}<N^{-1} \text { and }\left|f_{n}(u)\right| \leq 2|u|^{-1}
$$

The last inequality follows from $\left|\int_{x}^{y} e^{i u t} d t\right| \leq 2|u|^{-1}$. Setting $C(u)$ $=\max \left|f_{n}(u)\right|$, we cast the sum into the shape treated in paragraph 2 , except for a factor $p^{-1}$. The inequality in question is thus $\left|\sum\left(p-\xi_{n}\right) f_{n}(u)\right|$ $<\varepsilon p(1+u)^{\frac{1}{2}\left(\alpha^{2}-1\right)}$, where $B=C(u), \sigma^{2}=N C^{2}(u)$.

On the interval $0 \leq u \leq N$ we replace $\sigma^{2}$ and $B$ by their common upper bound $N^{-1}$, and choose $Y=\varepsilon p N^{\frac{1}{\left.2^{(\alpha 2}-1\right)}}$. Then $Y B<p \sigma^{2}$ and we obtain an exponential bound $4 \exp -c p^{-1} \sigma^{-2} Y^{2}$. Here the exponent exceeds $c p N N^{\alpha^{2}-1}>N^{\delta}$ because $-\beta^{2}+1+\alpha^{2}-1>0$. When $u>N$ we use $B=2 u^{-1}, \sigma^{2}=4 N u^{-2}, Y=\varepsilon p u^{\frac{1}{2}}\left(\alpha^{2}-1\right)$. To choose the best value of $t$ in Chebyshev's inequality we must verify that $Y B<2 p \sigma^{2}$, and this is true if $u^{\alpha^{2}+1}<N^{2}$. The exponent obtained exceeds $c p^{-1} \sigma^{-2} Y^{2}>p N^{-1} u^{\alpha^{2+1}} \geq N^{\delta}$, as before. For the remaining numbers $u$, defined by the inequality $u^{\alpha+1}>N^{2}$, we choose $t=\eta B^{-1}$ with a small constant $\eta>0$ and obtain from Chebyshev's inequality a bound $4 \exp -c B^{-1} Y$, wherein $B^{-1} Y>N^{\delta}$.

Thus, for each individual $u \geq 0$ the inequality sought holds except on a set of measure exp $-N^{\delta}$; in particular, at $u=0,\left\|m_{1}\right\|<2$, except on such a set. Thus, with probability near 1 , the result is valid for fractions $u=j N^{-2}, 0 \leq j \leq N^{4}$, and since $\hat{m}_{1}-\hat{m}_{0}$ has derivative at most 2 , this disposes of the interval $0 \leq u \leq N^{2}$ (since the error introduced 
by passing to real numbers $u$ doesn't exceed $N^{-2}$ ). When $u>N^{2}$, we use the inequality

$$
\left|\hat{m}_{1}(u)\right|<2 u^{-1} p^{-1} \sum \xi_{n}<4 u^{-1} N=o\left(u^{-\frac{1}{2}}\right) .
$$

An approach more congenial in Fourier analysis is to prove the inequality for all integers $k$ and then pass to real numbers $u$ by expanding $e^{i u t}$ in terms of $e^{i k t}$, on the interval $0 \leq t \leq 1$, whose length is less than $2 \pi$.

Using the lemma carefully we can prove Theorem 1 . Once $\eta$ is specified in $\left(0, \frac{1}{2}\right)$ we state once and for all that the $n$-th step, in the inductive process to be described, must be accomplished except on a set of $P<\eta+\cdots+\eta^{n}$, and the measures constructed in the $n$-th step must have mass $m$ in the interval $|1-m|<\eta+\cdots+\eta^{n}$. In an obvious way we make $\beta-2 b^{\frac{1}{2}}$ increase to $\alpha$. The lemma is applied first with $(r, s)=(0,1)$, and the random measures $m_{1}$ constructed are step-functions, with level intervals $j N^{-1}(0 \leq j<N)$. As $N$ is fixed, we can apply the lemma to these $N$ intervals $(r, s)$, and then sum the $N$ different measures constructed to obtain a random measure $m_{2}$ such that $\left|\hat{m}_{2}(t)-\hat{m}_{1}(t)\right|$ $<1 / 4(1+t)^{\frac{1}{2}\left(\alpha^{2}-1\right)}$ for all $t>0$. The closed support of $m_{2}$ is contained in that of $m_{1}, \cdots$. Clearly we can find a limit measure $\mu$, of mass

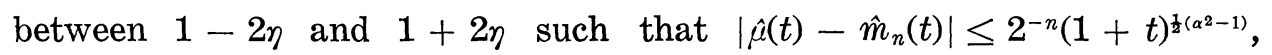
supported in a compact subset of $E_{\alpha}$. Since $\mu$ is defined except on a set of $P<2 \eta$, Theorem 1 is completely proved.

4. In the proof of Theorem 2 we require a lemma somewhat analogous to the one already proved; Fourier transforms are of little use here, since the set $F$ need not carry any measure whose transform $\hat{\mu}$ tends to zero. We therefore work directly on the metrical properties of measures, assigning to each measure $\mu_{0}$ on $[0,1]$ a random measure $\mu_{1}$ by the same process as before. Let $0 \leq \beta<\alpha<1, \alpha^{2}<d \leq 1$.

LEMMA. Suppose that $\mu_{0}(I) \leq C|I|^{d}$ for all intervals $I$ of length $|I|$. Then the inequality $\left|\mu_{0}(I)-\mu_{1}(I)\right| \leq \varepsilon|I|^{d-\alpha^{2}}$ for all intervals $I$ holds, with probability approaching 1 as $N \rightarrow \infty$.

Proof. Because $\mu_{1}(S) \leq p^{-1} \mu_{0}(S)$ for all sets $S$, the inequality is valid for intervals $I$ so small that $2 p^{-1} C|I|^{d}<\varepsilon|I|^{d-\alpha^{2}}$, or $|I|^{\alpha^{2}}<\varepsilon^{\prime} p$. Now $p=N^{-\beta^{2}} N^{o(1)}$, so that the upper bound on $|I|$ exceeds $N^{-1}$ for large $N$. For larger intervals we have the partition of $\mu_{0}$ and $\mu_{1}$ determined 
by $q$, and as before we omit the superscript. Let then $I \subseteq(0,1)$ and observe that

$$
\mu_{0}(I)-\mu_{1}(I)=p^{-1} \sum\left(p-\xi_{n}\right) \mu_{0}\left(I \cap I_{n}\right) .
$$

Now $\mu_{0}\left(I \cap I_{n}\right) \leq C N^{-d}$, while $\sigma^{2} \leq C N^{-d} \mu_{0}(I) \leq C^{\prime} N^{-d}|I|^{d}$. To estimate the probability of the event $\left|\sum\right|>\varepsilon p|I|^{d-\alpha^{2}}$ we use the exponential integrals with $t=\eta N^{d}\left(\eta>0\right.$ small). Here $t Y$ majorizes $t^{2} p \sigma^{2}$ because $|I| \leq 1$; moreover $t Y>c N^{d}|I|^{d-\alpha^{2}} p>N^{\delta}$ because $|I| \geq N^{-1}$ and $\alpha^{2}>\beta^{2}$. This estimate is strong enough to account for the $N^{2}$ intervals $I$ composed of adjacent intervals $I_{n}$; because the $\mu_{1}$-measure of an interval of length $N^{-1}$ is at most $C p^{-1} N^{-a}=o\left(N^{\alpha^{2}-d}\right)$, this in turn accounts for all intervals of length $|I| \geq N^{-1}$; now the lemma is completely proved:

To prove the first statement in Theorem 2, let $\operatorname{dim} F>e \geq \alpha^{2}$, so that $F$ carries a measure $\mu_{0}$ subject to a Lipschitz condition with exponent $e$ [3, Ch. III]. The lemma can then be applied to construct a sequence of measures $\mu_{N}$, concentrated in $F$, whose limit measure is concentrated in $F \cap E_{\alpha}$ and has mass $>\frac{1}{2}$; each $\mu_{N}$ fulfills a Lipschitz condition with exponent $e$, while the entire sequence fulfills a uniform (with respect to $N$ ) Lipschitz condition with exponent $e-\alpha^{2}$, ensuring that $F \cap E_{\alpha}$ has dimension at least $e-\alpha^{2}$. As before, this can be accomplished on a set of probability arbitrarily close to 1 , so $P\left\{\operatorname{dim} F \cap E_{\alpha}\right.$ $\left.\geq e-\alpha^{2}\right\}=1$.

To prove the remaining statements in Theorem 2 we choose for $F_{1}$ and $F_{2}$ certain dyadic sets, defined as follows. To each strictly increasing sequence $M=\left(m_{k}\right)$ of positive integers we associate the set of all infinite sums $\sum \varepsilon_{k} 2^{-m_{k}}\left(\varepsilon_{k}=0,1\right)$. The Hausdorff dimension of $F$ is then $\lim \inf k / m_{k}$, the lower density of $M$ [2, Ch. II]. For $\mathrm{F}_{1}$ we choose $m_{k}=\left[d^{-1} m_{k}\right]$, so that $m_{k+1}>m_{k} \geq 1$ and $M$ has density $d \geq 1-\alpha^{2}$. Each integer $k \geq 1$ determines a covering of $F_{1}$ by $2^{k}$ intervals $J$ of length $2^{-m_{k}}$; let us estimate the number of intervals $J$ that contain a number $t$, for which $|X(t+h)-X(t)|>\beta g(h)$, for some number $h$ in the range $2^{-m_{k}} \leq h \leq k 2^{-m_{k}}$. The expected number is at most $2^{k} 2^{-\beta^{2} m_{k}} 2^{o(k)}$, and it is almost sure that for large $k$ a bound of this type is valid. Clearly this implies that $\operatorname{dim} F_{1} \cap E_{\alpha} \leq d-\beta^{2}$ (whenever $\beta<\alpha^{2}$ ) hence $P\left\{\operatorname{dim} F_{1} \cap E_{\alpha} \leq d-\alpha^{2}\right\}=1$. Moreover, when $d<\alpha^{2} F_{1} \cap E_{\alpha}=\phi$ almost surely.

We now sketch briefly a curious result about the critical case $d=\alpha^{2}$, 
choosing a sequence $M$ with $m_{k}=d^{-1} k+o(k), m_{k}-d^{-1} k \rightarrow \infty$. As will be explained below, $F_{1}$ carries a measure $\mu$ satisfying the Lipschitz condition in each exponent $d_{1}<d$. Adapting the second lemma we can prove that $F_{1} \cap E_{\alpha}$ almost surely supports a continuous measure and must then be uncountable; a proper choice of $M$, taking account of the distribution of $S$, yields a set $F_{1}$ of dimension $\alpha^{2}$ such that

$$
|X(t+h)-X(t)| \leq \alpha g(h) \quad \text { for } h<h_{0} \text { and all } t \text { in } F_{1}
$$

almost surely. (The argument in this paragraph is adapted from [5].)

The sequence $M$ defining $F_{2}$ is described in terms of its counting function $v: v(s)=k$ if $m_{k} \leq s<m_{k+1}$. We require that $d=1-\alpha^{2}$ and that

(1) $v(s) \geq d s+s^{1 / 2}$ for $s \geq s_{0}$,

(2) $\liminf s^{-1} v(s)=d$,

(3) $v\left(t^{6}\right) \geq t^{6}-t$ for all integers $t$ in an infinite set $T$.

Then $F_{2}$ carries a product measure $\mu_{0}$ derived from its representation as a Cantor set; its modulus of continuity $w(h)=\sup \mu_{0}(a, a+h)$ is governed by the inequalities $w\left(2^{-s}\right) \leq 2 \cdot 2^{-v(s)}$.

Now we follow the proof of the first statement, setting $N=2^{t^{3}}$ for some $t$ in $T$. The inequality necessary for one step in the construction is $\left|\mu_{0}(I)-\mu_{1}(I)\right| \leq \varepsilon|I|^{d}$ for all intervals $I$. First of all $w(h)=o\left(h^{d}\right)$; thus $w(h) \leq \varepsilon p h^{d}$ for $h<N^{-1}$. In fact, for $h \leq 2^{-t^{6}}$, say $h=2^{-s}, w(h) h^{-d}$ $\leq 2^{-s^{1 / 2}}$, while $p>N^{-\beta^{2}} N^{o(1)}$, so the inequality $s \geq t^{6}$ yields $w(h) h^{-d} \leq N^{-1}$ $=o(p)$. For $h>2^{-t 6}$ we use the elementary inequality $w(h) \leq 2 h \cdot 2^{t 6} w\left(2^{-t^{6}}\right)$ $<4 h 2^{t}$. Also, $4 h 2^{t}<\varepsilon p h^{d}$ when $h \leq N^{-1}$, because $d+\alpha^{2}=1$. Thus we have disposed of intervals $I$ of length $<N^{-1}$.

For remaining numbers $h \geq N^{-1}$ we study sums $p^{-1} \sum \equiv p^{-1} \sum(p$ $\left.-\xi_{n}\right) \mu\left(I \cap I_{n}\right)$; in $\sum$ we have $B \leq w\left(N^{-1}\right)$ and $\sigma^{2} \leq w(h) w\left(N^{-1}\right)$. In the exponential integrals we take $t=\eta w^{-1}\left(N^{-1}\right)$ with a small $\eta>0$ and obtain a bound

$$
P\left\{\left|\sum\right|>\varepsilon p h^{d}\right\} \leq 2 \exp \eta^{2} p w(h) w^{-1}\left(N^{-1}\right) \exp -\varepsilon p h^{d} w^{-1}\left(N^{-1}\right) .
$$

Now $w(h)=0\left(h^{d}\right)$ so the exponent is negative for small $\eta$ and has modulus $>c p h^{d} w^{-1}\left(N^{-1}\right) \geq c p N^{-d} w^{-1}\left(N^{-1}\right)>N^{\delta}$ for a certain $\delta>0$; these inequalities are sufficient to construct a measure on $F_{2} \cap E_{\alpha}$ with modulus of continuity $0\left(h^{d}\right)$; so $F_{2} \cap E_{\alpha}$ has dimension $1-\alpha^{2}$.

By the same method we can prove an even stronger property for a set $F_{3}$ of dimension $1-\alpha^{2}$. Let $S$ be a sequence of positive numbers 
tending to 0 , and let $E_{\alpha}(S)$ be defined by the functional $\lim \sup X(t+h)$ $-X(t) / g(h), h \in S$. Then $F_{3}$ will be a compact set of dimension $1-\alpha^{2}$, and $\operatorname{dim} F_{3} \cap E_{\alpha}(S)=1-\alpha^{2}$ almost-surely, for each fixed null sequence S.

$F_{3}$ is a "compound" dyadic set, slightly more complicated than $F_{1}$ and $F_{2}$ in structure. Using the dyadic representation as before, we have sets $D_{q}$ defined as follows: $x=\sum \varepsilon_{k} 2^{-k}$ is in $D_{k}$ if either $\varepsilon_{k}=0$ on $q \leq k \leq d^{-1} q$, or $\varepsilon_{k}=0$ on $q^{2} \leq k \leq d^{-1} q^{2} ; F_{3}$ is the intersection $\cap D\left(q_{j}\right)$, where $q_{j+1}>(j+1) q_{j}^{2}$. Since each $D(q)$ has an efficient covering by dyadic intervals, $\operatorname{dim} F_{3} \leq 1-\alpha^{2}=d$. Each sequence of symbols $a_{j}=I$ or $I I$ determines a dyadic set contained in $F_{3}$ : when $a_{j}=I$ we take the first alternative allowed in $D\left(q_{j}\right)$, and the second when $\alpha_{j}=I I$. When the sequence $s=h_{1}>h_{2}>\ldots>h_{m}>\ldots$ is specified, there is a subsequence of $S$, say $\left(h_{m}^{*}\right)$ and a choice of the symbols $\alpha_{j}=I, I I$ with this property: the numbers $-\log h_{m}^{*}$ and the digits $\varepsilon_{k}$ omitted from the dyadic set become far apart in the sense that for large $m$ all integers $k$ in $\left[-\varepsilon \log h_{m}^{*},-\varepsilon^{-1} \log h_{m}^{*}\right]$ are unrestricted. Now, choosing the product measure on this special subset of $F_{3}$ and using $N \cong h_{m}^{-1}$ in the construction leading to $F_{2}$, we can construct a subset of $F_{3} \cap E_{\alpha}(S)$ of dimension $1-\alpha^{2}$.

\section{REFERFNCES}

[1] N. K. Bari, A treatise on trigonometric series, II. (Transl. of Russian) New York, 1964.

[2] J.-P. Kahane, Some random series of functions. Heath, Lexington, Mass., 1968.

[ 3 ] J.-P. Kahane et R. Salem, Ensembles parfaits et séries trigonométriques. Hermann, Paris, 1968.

[4a] R. Kaufman, Une propriété métrique du mouvement brownien. C. R. Acad. Sci. Paris 268A (1969), 727-728.

[4b] On the zeroes of some random functions. Studia Math. 34 (1970), 291-297.

[4c] Brownian motion and dimension of perfect sets. Canad. J. Math. 22 (1970), 674680.

[4d] A metric property of some random functions. Bull. Soc. Math. de France 99 (1971), 241-245.

[4e] Measures of Hausdorff-type, and Brownian motion. Mathematika, 19 (1972), 115-119.

[ 5 ] S. Orey and S. J. Taylor, How often on a Brownian path does the law of iterated logarithm fail? Pre-print, Minnesota, 1972.

[6] A. Zygmund, Contribution à l'unicite du développement trigonométrique. Math. Zeitsch. 24 (1926), 40-46. 